INPLASY

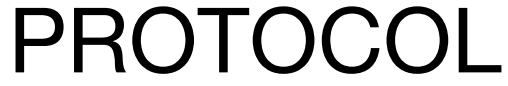

To cite: Lu et al. Effect of trigger point intervention on postoperative pain. Inplasy protocol 202140024. doi: 10.37766/inplasy2021.4.0024

Received: 05 April 2021

Published: 05 April 2021

Corresponding author: Wei Lu

luwei@shutcm.edu.cn

Author Affiliation:

Shanghai University of

Traditional Chinese Medicine

Support: Longhua Key Project.

Review Stage at time of this submission: Preliminary searches.

Conflicts of interest:

None declared.

\section{Effect of trigger point intervention on postoperative pain}

\author{
Lu, W1; Yu, Y2; Yao, R33 Li, J4.
}

Review question / Objective: With gradually social modernization and continuous aging of the population, the number of surgeries is marked increasing worldwide. However, oceans of studies indicated that poor control of postoperative pain is common all over the world. Commonly, postoperative pain can not be well managed and eliminated even in the most modern country. Importantly, poor postoperative pain control often leads to poor outcomes to patients, including long duration, sleep disorders, somnolence related syndromes, cardiopulmonary and thromboembolic complications. Therefore, it is very important to control the postoperative pain. Trigger point is a hyperirritable spot within a taut band of skeletal muscle that is painful on compression, stretch, overload, or contraction of the tissue which usually responds with a referred pain that is perceived distant from the spot. Because the trigger point is such an important intersection of sensory and motor functions, it can not only be a predictor of postoperative pain, but also a target for treatment of postoperative pain. Many researcher applied the trigger point intervention for the postoperative pain treatment, such as trigger point dry needing or trigger point injection with some. However, the efficacy of trigger point intervention on postoperative pain treatment was not totally discussed and the controversy always exists up to now.

INPLASY registration number: This protocol was registered with the International Platform of Registered Systematic Review and Meta-Analysis Protocols (INPLASY) on 05 April 2021 and was last updated on 05 April 2021 (registration number INPLASY202140024).

\section{INTRODUCTION}

Review question / Objective: With gradually social modernization and continuous aging of the population, the number of surgeries is marked increasing worldwide. However, oceans of studies indicated that poor control of postoperative pain is common all 
over the world. Commonly, postoperative pain can not be well managed and eliminated even in the most modern country. Importantly, poor postoperative pain control often leads to poor outcomes to patients, including long duration, sleep disorders, somnolence related syndromes, cardiopulmonary and thromboembolic complications. Therefore, it is very important to control the postoperative pain. Trigger point is a hyperirritable spot within a taut band of skeletal muscle that is painful on compression, stretch, overload, or contraction of the tissue which usually responds with a referred pain that is perceived distant from the spot. Because the trigger point is such an important intersection of sensory and motor functions, it can not only be a predictor of postoperative pain, but also a target for treatment of postoperative pain. Many researcher applied the trigger point intervention for the postoperative pain treatment, such as trigger point dry needing or trigger point injection with some. However, the efficacy of trigger point intervention on postoperative pain treatment was not totally discussed and the controversy always exists up to now.

Condition being studied: The efficacy of trigger point intervention on postoperative pain treatment will be reviewed and metaanalysis will be performed.

\section{METHODS}

Search strategy: We will conduct literature searches on these following electronic databases: Pubmed, the Cochrane Library, Exceerpta Medica Database (EMBASE), Web of Science, Physiotherapy evidence database. Databases were retrieved from the earliest data available to $2020 / 8 / 30$. The search strategies were composed of such items: ("post operative") AND ("pain") AND ("trigger point"). The references of previous literatures concerning trigger point on post operative pain were tracked carefully. http://www.clinicaltrial.gov, http:// www.chictr.org.cn/index.aspx and http:// www.who.int/trialsearch will be searched to investigate any ongoing clinical trials. The reference lists from relevant systematic reviews, meta-analysis and included primary studies will be searched.

Participant or population: Subjects suffers pain after surgery.

Intervention: Trigger point needling or injection.

Comparator: The placebo or usual care which includes standard medications, physiotherapy and other normal care.

Study designs to be included: Randomized controlled trial or non-randomized controlled trial, before and after, interrupted time series, cross-over trail, observational studies.

Eligibility criteria: (1) The trails contained the subjects with definitely diagnosis of post operative pain. (2) The intervention is based on trigger point.

Information sources: We will conduct literature searches on these following electronic databases: Pubmed, the Cochrane Library, Exceerpta Medica Database (EMBASE), Web of Science, Physiotherapy evidence database. The references of previous literatures concerning trigger point on post operative pain were tracked carefully. http:// ww w. clinicaltrial.gov, http:/ / www.chictr.org.cn/index.aspx and http:// www.who.int/trialsearch will be searched to investigate any ongoing clinical trials. The reference lists from relevant systematic reviews, meta-analysis and included primary studies will be searched.

Main outcome(s): VAS or Numeric rating scores.

Quality assessment / Risk of bias analysis: The Physiotherapy Evidence Database (PEDro) was used to assess the quality of included articles. The methodological criteria were scored as: Yes (one point), No (zero points) or Don't know (zero points). The PEDro score of each selected study provided an indicator of the methodological quality $(9-10=$ excellent; $6-8=$ good; $4-5=$ fair; $<4=$ poor). 
Strategy of data synthesis: A narrative and qualitative synthesis of included studies was performed. Review Manager (Revman, Version 5.3) software provided by the Cochrane Collaboration was used for data analysis. For continuous variables, standardized mean difference (SMD) and 95\% confidence interval $(\mathrm{Cl})$ were used for statistics. The heterogeneity tests of each outcome were performed using Chisquared test and 12 statistic. When no significant heterogeneity was observed (12 $<50 \%$ ), the fixed-effects model was used to perform meta-analysis. When heterogeneity was detected $(P<0.05$ and 12 $\geq 50 \%$ ), a random-effects model was used. Funnel plots were planned to visually investigate the asymmetry and potential publication bias along with the quantitatively Egger test. The subgroup and sensitivity analyses were also planned to explore possible reasons for statistical heterogeneity when $12>50 \%$.

Subgroup analysis: If available, subgroup analyses will be performed following below items in our plan: type of surgery, type of intervention (Needling or injection), male/ female of subjects, location of study, duration of treatment, follow up times.

Sensitivity analysis: Sensitivity analysis will by used by removing one by one or statistical change when $12 \geq 50 \%$.

Language: None limited.

Country(ies) involved: Mainland China.

Keywords: Postoperative pain; trigger point; meta analysis.

Contributions of each author:

Author 1 - Wei Lu.

Email: luwei@shutcm.edu.cn

Author 2 - Yang Yu.

Email: 13661931708@163.com

Author 3 - Ruoyu Yao.

Email: yaoruoyu@live.com

Author 4 - Jiong Li.

Email: joe8989@shutcm.edu.cn 